\title{
Análisis del control dinámico de un tren de destilación multicomponente del área de recuperación de solvente de una planta de polietileno lineal
}

\author{
Dynamic control analysis of a multicomponent distillation train of the \\ solvent recovery area of a linear polyethylene plant
}

\author{
Gustavo Enrique Ramos Montero. \\ Laboratorio de Ingeniería Química, Universidad del Zulia. Maracaibo, Venezuela. \\ Correo:geramos@fing.luz.edu.ve \\ Charles Albert Gutiérrez Mendoza. \\ Laboratorio de Ingeniería Química, Universidad del Zulia. Maracaibo, Venezuela. \\ Correo: chgutierrez@fing.luz.edu.ve \\ Beatriz María Marrufo Pachano. \\ Laboratorio de Ingeniería Química, Universidad del Zulia. Maracaibo, Venezuela. \\ Correo:bmarrufo@fing.luz.edu.ve
}

Recibido: 9 de marzo 2019

Aceptado: 17 de julio 2019

\begin{abstract}
Resumen
Esta investigación aporta el análisis dinámico de un tren de destilación, que en lugar del tradicional estudio de una mezcla binaria, este se realizó para una mezcla multicomponente del área de recuperación de solvente de una planta de polietileno lineal. Esto se llevó a cabo a través de una simulación dinámica en Aspen Plus® con la finalidad de predecir el comportamiento de las variables operacionales (temperatura, presión, flujo, nivel o composición) al momento de ocurrir una interrupción del flujo de alimentación al área de destilación para modelar una parada del área de reacción, y definir los procedimientos que compensen las desviaciones en las variables controladas. Se evaluaron varios métodos termodinámicos: Soave-Redlich-Kwong, Peng-Robinson, Lee-Kesler-Plock, Soave-Redlich-Kwong-Boston-Mathias y Peng-Robinson-Boston-Mathias; se eligió el método SRKBM, ya que presenta menores porcentajes de error en la mayoría de las propiedades estimadas. Esta comparación rigurosa le añade mayor confiabilidad a los valores reportados en las simulaciones. Los resultados mostraron una desestabilización de los controladores de nivel de las columnas de destilación A y B, además de un aumento de $14{ }^{\circ} \mathrm{C}$ aproximadamente en el perfil de temperaturas de la columna A. La columna $\mathrm{C}$ no presentó desviaciones de sus variables operacionales. Finalmente, para estabilizar el sistema fue necesario realizar tres pasos que involucran el cambio a modo manual y del porcentaje de apertura de la válvula de los controladores de flujo de vapor a la columna A (50\%), de presión del separador D-101 (50\%) y de flujo de tope de la columna B (43\%).
\end{abstract}

Palabras clave:

Simulación, modelaje, control de procesos, dinámica, lazos de control 


\begin{abstract}
This research provides the dynamic analysis of a distillation train, which instead of the traditional study of a binary mixture, this was carried out for a multicomponent mixture of the solvent recovery area of a linear polyethylene plant. This was done through a dynamic simulation in Aspen Plus ${ }^{\circledR}$ with the purpose of predicting the behavior of the operational variables (temperature, pressure, flow, level or composition) when an interruption of the feed flow occurs in the distillation area to model a reaction area shutdown, and then establish procedures that compensate for deviations in the controlled variables. Several thermodynamic methods were evaluated: Soave-Redlich-Kwong, Peng-Robinson, Lee-Kesler-Plock, PengRobinson-Boston-Mathias y Soave-Redlich-Kwong-Boston-Mathias; the SRKBM method was chosen, since it presents lower error percentages in most of the estimated properties. This rigorous comparison adds greater reliability to the values reported in the simulations. The results showed a destabilization of the level controllers of distillation columns $\mathrm{A}$ and $\mathrm{B}$, in addition to an increase of approximately $14{ }^{\circ} \mathrm{C}$ in the temperature profile of column A. Column C did not show deviations from its operational variables. Finally, to stabilize the system, it was necessary to carry out three steps involving the switch to manual mode and changing the valve opening percentage of the column A steam flow controller (50\%), the D-101separator pressure (50\%) and the column B top flow controller (43\%).
\end{abstract}

Keywords:

Simulation, modeling, process control, dynamics, control loops 


\section{INTRODUCCIÓN}

El polietileno lineal de baja densidad (PELBD) ha sido ampliamente utilizado en la vida cotidiana, como material de embalaje o piezas de automóviles (Kusolsongtawee y Bumroongsri, 2017) debido a que tiene una mejor resistencia mecánica y procesabilidad en comparación con el polietileno de alta densidad, causado por la presencia de ramificaciones cortas (Sabetzadeh, Bagheri y Masoomi, 2016). Típicamente, el PELBD se obtiene por copolimerización de etileno con $\alpha$-olefinas como comonómeros con catalizadores de Ziegler-Natta (Gagieva et al., 2015). La densidad de este polímero varía entre 915 y $930 \mathrm{~kg} / \mathrm{m}^{3}$ mediante el uso de distintos comonómeros como lo son: 1-buteno, 1-hexeno o 1-octeno. Este proceso en solución opera entre $160-220{ }^{\circ} \mathrm{C}$ y 3.4-34 MPa; bajo estas condiciones el polímero es disuelto en el solvente, comúnmente ciclohexano (Malpass, 2010).

La planta de polietileno lineal en estudio en esta investigación tiene como finalidad producir resinas lineales de baja densidad. Para esto, el proceso cuenta con las áreas de reacción, purificación, acabado y servicios; asimismo, utiliza una tecnología en donde el reactor es alimentado con etileno como monómero y el comonómero (ya sea 1-buteno, 1-octeno o una mezcla de estos) con ciclohexano como solvente.

Como en la mayoría de las plantas químicas, el área de reacción de una planta de polietileno lineal representa el núcleo del proceso productivo, por lo que su correcto funcionamiento es fundamental. No obstante, es necesario realizar frecuentemente paradas de sus distintos procesos. Esto trae como consecuencia que las áreas subsecuentes que dependen del área de reacción, deban adaptarse para seguir funcionando a pesar de suspender la operación de alguno de sus procesos (Whiteley, 2012). Estas secciones incluyen el área de recuperación de solvente que se encarga de separar y purificar cada uno de los componentes una vez terminada la polimerización.

Durante este periodo, es necesario responder rápidamente a los requerimientos del proceso mediante la manipulación de las variables, ya que esto contribuye a la eficiencia y seguridad de la planta para poder volver a operar normalmente en el menor tiempo posible una vez restablecido el servicio en el área de reacción (Fabro, Arruda y Neves, 2005). Estos procedimientos de parada pueden ser mejorados con la ayuda de la automatización y control de procesos. Las ventajas de incluir procedimientos automáticos residen en reducir el tiempo de respuesta, incrementa la precisión y repetitividad ya que minimiza el error humano (Leitao, P., Colombo W. y Karnouskos S., 2015).

Actualmente, la sección de síntesis del polímero de la planta de polietileno lineal de baja densidad cuenta con una secuencia de parada del área de reacción, este procedimiento involucra la interrupción del suministro de 1-buteno e hidrógeno, adecuación de intercambiadores de calor, ajuste de presión en los separadores, entre otros pasos.

Sin embargo, el área de recuperación de solvente, que incluye tres columnas de destilación, no cuenta con un procedimiento automatizado que permita una respuesta inmediata al momento de detener la reacción. Esto, teniendo en cuenta que las columnas de destilación son unidades complejas que exhiben dinámicas no lineales debido a sus relaciones de equilibrio no ideales y configuraciones 
de operación (Abdullah, Ahmad y Aziz, 2009); así como también, a las interacciones entre las unidades causadas por la integración del calor y recirculación de corrientes (Xu y Bao, 2017), y la necesidad de alcanzar alta pureza de sus productos; hace necesario la implementación de una secuencia automática de control que permita monitorear y regular variables como temperatura, presión, flujo, nivel y composición en las columnas de destilación de manera segura y rápida con la finalidad de alcanzar condiciones estables y requerimientos en las especificaciones con el menor consumo de energía e insumos al momento de ocurrir una parada de la sección de reacción. El arranque y paro programado de la sección de recuperación de solvente requieren de perfecta sincronización de eventos en secuencia que ameritan el uso de computadoras e instrumentos de medición en línea para evitar accidentes y daños en los equipos (Batres, 2013).

La simulación dinámica es ampliamente utilizada para evaluar sistemas de control y automatización, para regular la operación de un proceso cercano a su estado estable. Además, permite predecir el comportamiento de las variables al ocurrir alguna emergencia o falla en los equipos. Y así poder establecer distintos procedimientos para compensar los cambios de las condiciones normales de operación (Luyben, 2012).

Recientes investigaciones de modelaje y simulación dinámica de procesos de destilación se enfocan en mezclas binarias (Taqvi, Tufa y Muhadizir, 2016), mezclas multicomponentes (Niculescu, et al., 2017) o trenes de destilación (Hosgor, et al., 2014). Sin embargo, pocas investigaciones involucran trenes de destilación de sistemas multicomponentes en estado dinámico, de allí la relevancia de esta investigación.

Debido a lo anterior, se estudia mediante el uso de Aspen Plus ${ }^{\circledR}$ y Aspen Dynamics ${ }^{\circledR}$, el área de recuperación de solvente, el cual incluye tres columnas de destilación, cuya función es separar el etileno, buteno, ciclohexano y oligómeros formados durante la reacción. Con el objetivo de proponer un procedimiento que permita una respuesta inmediata al momento de presentarse una parada del área de reacción, y sea capaz de monitorear y regular variables: temperatura, presión, flujo, nivel y composición en las columnas de destilación de modo seguro y rápido de tal manera de alcanzar condiciones estables.

\section{METODOLOGÍA}

\subsection{Definición de los componentes del sistema}

Para iniciar el proceso de simulación en estado estacionario se tomaron como referencia los componentes descritos en los balances de masa por diseño de plantas de producción de polietileno lineal; estos son: etileno $\left(\mathrm{C}_{2} \mathrm{H}_{4}\right)$ como monómero, ciclohexano $\left(\mathrm{C}_{6} \mathrm{H}_{12}\right)$ como solvente, 1-buteno $\left(1-\mathrm{C}_{4} \mathrm{H}_{8}\right)$ como comonómero, 2-buteno $\left(2-\mathrm{C}_{4} \mathrm{H}_{8}\right)$ producto de la isomerización del 1-buteno durante la reacción y undecano $\left(\mathrm{C}_{11} \mathrm{H}_{24}\right)$ para modelar los oligómeros.

\subsection{Selección del modelo termodinámico}

Una vez definidos los componentes del sistema, se tomó en cuenta la naturaleza de los mismos y las condiciones de temperatura y presión del sistema para realizar un filtrado preliminar de los métodos termodinámicos a seleccionar para la reproducción del comportamiento PVT del sistema 
(Carlson, 1996). Posteriormente, se llevó a cabo un estudio comparativo que permitió seleccionar el método termodinámico que se adapte más eficientemente a las propiedades de las corrientes y reproduzca el comportamiento PVT del sistema. En este sentido, se compararon los métodos Soave-Redlich-Kwong (SRK), Peng-Robinson (PR), Lee-Kesler-Plock (LKP), Peng-RobinsonBoston-Mathias (PRBM) y Soave-Redlich-Kwong-Boston-Mathias (SRKBM). Las propiedades comparadas fueron el flujo de las fases, densidad, calor específico y viscosidad disponibles dentro de la documentación de la planta. Se seleccionó la corriente 5 de alimentación al D-101, ya que esta es una corriente de mezcla vapor-líquido, Figura 1.

\subsection{Descripción del proceso}

La alimentación del proceso consiste en un flujo de mezcla de $\mathrm{C}_{6} \mathrm{H}_{12} \mathrm{y} \mathrm{C}_{11} \mathrm{H}_{24}$ (corriente 1) y un flujo de $\mathrm{C}_{2} \mathrm{H}_{4}, 1-\mathrm{C}_{4} \mathrm{H}_{8}$ y $2-\mathrm{C}_{4} \mathrm{H}_{8}$ (corriente 2) que se combinan para luego ser enviados tanto a la columna A como al intercambiador de calor H-101 y a su vez al tanque de retención D-101. Además, este tanque es alimentado por un flujo de isómeros de $\mathrm{C}_{4} \mathrm{H}_{8}$ y $\mathrm{C}_{6} \mathrm{H}_{12}$ (corriente 4) proveniente de la zona de reacción. La primera columna (A) separa principalmente el $\mathrm{C}_{2} \mathrm{H}_{4}, 1-\mathrm{C}_{4} \mathrm{H}_{8}$ y $2-\mathrm{C}_{4} \mathrm{H}_{8}$ por el

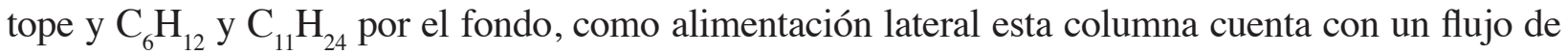
isómeros de $\mathrm{C}_{4} \mathrm{H}_{8}$ (corriente 9), la segunda columna (B) permite separar el $\mathrm{C}_{6} \mathrm{H}_{12}$ del $\mathrm{C}_{11} \mathrm{H}_{24}$, el fondo de esta columna se envía a la última columna $(\mathrm{C})$ que sirve como extensión de la segunda columna para terminar de separar el $\mathrm{C}_{11} \mathrm{H}_{24}$ y recircular el $\mathrm{C}_{6} \mathrm{H}_{12}$, como se muestra en la Figura 1.

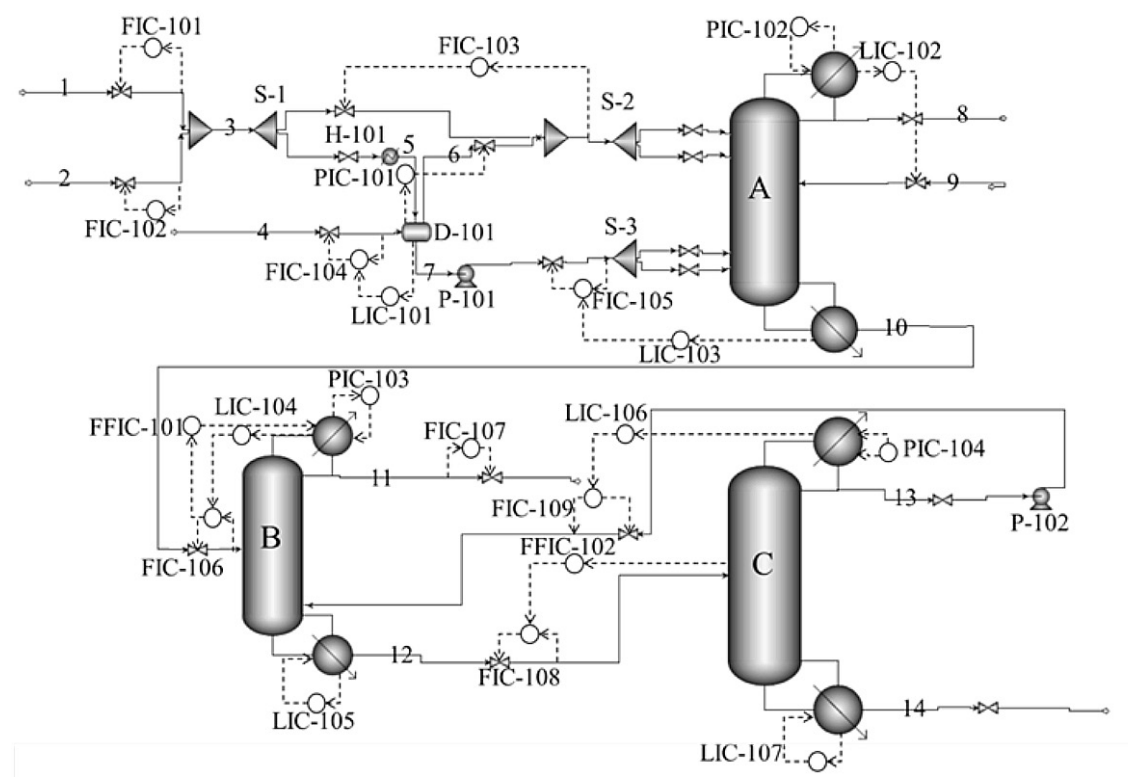

Figura 1. Diagrama de la simulación del área de recuperación de solvente

\subsection{Desarrollo del modelo mediante Aspen Plus}

Luego de definir los pasos del proceso, se procedió a realizar el modelaje de este. Las columnas fueron formadas mediante el uso del módulo Radfrac (Luyben, 2012 y 2013), el tanque se modeló como un separador Flash. Por su parte, para el intercambiador de calor se utilizó el modelo Heater, 
Tabla 1. Especificaciones de las columnas de destilación y equipos adicionales

\begin{tabular}{|c|c|c|c|}
\hline Columnas & A & B & $\mathrm{C}$ \\
\hline Etapas teóricas & 40 & 28 & 30 \\
\hline Etapa de alimentación & $\begin{array}{c}\text { Vapor: } 15 \text { y } 16 \\
\text { Líquido: } 20 \text { y } 21 \\
\text { Corriente } 9: 1\end{array}$ & $\begin{array}{c}\text { A: } 8 \\
\text { C: } 30\end{array}$ & B: 3 \\
\hline \multicolumn{4}{|c|}{ Geometría de los platos } \\
\hline Diámetro (mm) & 2500 & 2300 & 1000 \\
\hline Espaciado (mm) & 600 & 500 & 550 \\
\hline Altura del vertedero $(\mathrm{mm})$ & 40 & 55 & 40 \\
\hline \multicolumn{4}{|c|}{ Tambores de reflujo } \\
\hline Longitud (mm) & 5000 & 7000 & 3000 \\
\hline Diámetro (mm) & 2000 & 3000 & 1000 \\
\hline Condensador & \multicolumn{3}{|c|}{ Total } \\
\hline \multicolumn{4}{|c|}{ Rehervidores } \\
\hline Tipo & \multicolumn{3}{|c|}{ Horizontal/Elíptico } \\
\hline Longitud (mm) & 4500 & 5800 & 3600 \\
\hline Diámetro (mm) & 1300 & 1600 & 1000 \\
\hline \multicolumn{4}{|c|}{ Tanque de alimentación D-101 } \\
\hline Tipo & \multicolumn{3}{|c|}{ Horizontal/Elíptico } \\
\hline Longitud (mm) & \multicolumn{3}{|c|}{7000} \\
\hline Diámetro (mm) & \multicolumn{3}{|c|}{3000} \\
\hline
\end{tabular}

Tabla 2 Especificaciones de diseño de las corrientes de alimentación al área de destilación

\begin{tabular}{|c|c|c|c|c|c|c|c|c|}
\hline Corriente & \multicolumn{2}{|c|}{1} & \multicolumn{2}{|c|}{2} & \multicolumn{2}{|c|}{4} & \multicolumn{2}{|c|}{9} \\
\hline $\mathrm{T}\left({ }^{\circ} \mathrm{C}\right)$ & \multicolumn{2}{|c|}{243} & \multicolumn{2}{|c|}{243} & \multicolumn{2}{|c|}{155} & \multicolumn{2}{|c|}{37} \\
\hline $\mathrm{P}(\mathrm{MPa})$ & \multicolumn{2}{|c|}{2.65} & \multicolumn{2}{|c|}{2.65} & \multicolumn{2}{|c|}{2.6} & \multicolumn{2}{|c|}{2.98} \\
\hline Composición & $\mathrm{kg} / \mathrm{h}$ & $\% \mathrm{p} / \mathrm{p}$ & $\mathrm{kg} / \mathrm{h}$ & $\% \mathrm{p} / \mathrm{p}$ & $\mathrm{kg} / \mathrm{h}$ & $\% \mathrm{p} / \mathrm{p}$ & $\mathrm{kg} / \mathrm{h}$ & $\% \mathrm{p} / \mathrm{p}$ \\
\hline $\mathrm{C}_{6} \mathrm{H}_{12}$ & 78189.9 & 99.62 & - & - & 24730.8 & 92.39 & - & - \\
\hline $1-\mathrm{C}_{4} \mathrm{H}_{8}$ & - & - & 8300.6 & 76.52 & 625.2 & 2.36 & 2086.5 & 98.99 \\
\hline $2-\mathrm{C}_{4} \mathrm{H}_{8}$ & - & - & 1460.9 & 13.47 & 1340.4 & 5.14 & 21.1 & 1.01 \\
\hline $\mathrm{C}_{2} \mathrm{H}_{4}$ & - & - & 1085.9 & 10.01 & - & - & - & - \\
\hline $\mathrm{C}_{11} \mathrm{H}_{24}$ & 298.8 & 0.38 & - & - & - & - & - & - \\
\hline Total & 78488.7 & 100 & 10847.4 & 100 & 26696.4 & 100 & 2107.6 & 100 \\
\hline
\end{tabular}


las bombas y válvulas fueron hechas con los módulos Pump y Valve, respectivamente. En la Tabla 1 se presentan los datos de dimensiones y características de estos equipos. Por otro, en la Tabla 2 se visualizan los datos de las especificaciones de las corrientes de alimentación al modelo de simulación estudiado; y en la Tabla 3 las condiciones de operación. Cada uno de estos datos fueron obtenidos de la revisión de una planta de polietileno lineal de baja densidad.

Tabla 3. Especificaciones de diseño de las corrientes de alimentación al área de destilación

\begin{tabular}{cccc}
\hline Columna & $\mathrm{A}$ & $\mathrm{B}$ & $\mathrm{C}$ \\
\hline Relación de reflujo (másica) & 3.1 & 0.3 & 0.6 \\
Relación D/F (másica) & 0.1350 & 0.9319 & 0.9474 \\
Separadores & $\mathrm{S}-1$ & $\mathrm{~S}-2$ & $\mathrm{~S}-3$ \\
Fracción de separación & 0.80 & 0.54 & 0.69 \\
\hline Intercambiador de calor & & $\mathrm{H}-101$ & \\
Temperatura $\left({ }^{\circ} \mathrm{C}\right)$ & & 205 & \\
Presión (MPa) & & 2.5 & \\
Tanque de alimentación & & 211 & \\
Temperatura $\left({ }^{\circ} \mathrm{C}\right)$ & & 2.5 & P-102 \\
Presión $(\mathrm{MPa})$ & $\mathrm{P}-101$ & & 1.2 \\
\hline Bombas & 2.76 & & \\
\hline Presión de descarga (MPa) & & & \\
\hline
\end{tabular}

\subsection{Desarrollo del modelo de simulación en estado dinámico}

Una vez definido el modelo de simulación con datos reales del sistema, se procedió a generar el dinámico del proceso. Primero, se debe especificar la geometría de los equipos (Tabla 1), así como también se deben definir las principales válvulas de control asociadas al área de destilación (Figura 1).

\subsection{Migración del modelo en estado estacionario a estado dinámico con el simulador Aspen Plus Dynamics}

Al definir las válvulas necesarias para el sistema de control se realizó la migración al estado dinámico. Se definió una exportación de la simulación controlada por presión ( $P$ Driven Dyn Simulation) bajo las siguientes premisas: las presiones de alimentación y productos quedaron especificadas y los flujos fueron obtenidos a través de diferencias de presión. Cada uno de los lazos de control fue configurado dentro de la simulación dinámica. 


\subsection{Configuración de los lazos de control del área de recuperación de solvente}

Luego, al iniciar el modelaje en estado dinámico se describieron los lazos de control del área de recuperación de solvente, de acuerdo con su función y estructura, tomando en cuenta el equipo al cual están asociados y su relevancia en análisis dinámico.

En la Figura 1 se muestran la ubicación de los lazos de control utilizados de acuerdo con la distribución actual del sistema automático de control del área de recuperación de solvente de la planta de polietileno lineal de baja densidad.

A su vez, en la Tabla 4 fueron los parámetros de los controladores y la función de cada lazo; se utilizó el método de Ziegler-Nichols para entonar los controladores (Ziegler y Nichols, 1993), basado en la respuesta de la variable controlada del controlador en lazo abierto luego de realizar una perturbación de su variable manipulada.

\subsection{Análisis dinámico de las variables de proceso del área de recuperación de solvente}

Una vez establecida la simulación dinámica y garantizada su operación estable, se procedió a interrumpir el suministro de $\mathrm{C}_{2} \mathrm{H}_{4}$ e isómeros de $\mathrm{C}_{4} \mathrm{H}_{8}$ de alimentación a la sección de recuperación de solvente. Lo anterior se llevo a cabo cerrando completamente la válvula del controlador FIC-102, con la finalidad de modelar una parada de la sección de reacción en donde el flujo de monómeros y comonómeros se interrumpe y analizar el comportamiento de las variables de cada uno de los equipos. Finalmente, cabe mencionar que se tomaron en cuenta las siguientes variables: presión, nivel, temperatura y flujo, considerando su desviación de los valores de operación estable.

\subsection{Secuencia de pasos para la adecuación de la sección de recuperación de solvente}

Luego de realizar el análisis dinámico de las variables del proceso al interrumpir el suministro de $\mathrm{C}_{2} \mathrm{H}_{4}$ y $\mathrm{C}_{4} \mathrm{H}_{8}$, se estudiaron los pasos que deban llevarse a cabo para poder estabilizar las variables principales del área de recuperación de solvente. Se realizaron cambios en las variables manipuladas (apertura de válvulas o flujo) hasta estabilizar los lazos de control, comenzando por aquellos que se encuentren más alejados de su valor deseado.

\section{DISCUSIÓN DE RESULTADOS}

\subsection{Selección del método termodinámico}

Las corrientes en estudio están compuestas principalmente de $\mathrm{C}_{6} \mathrm{H}_{12}, \mathrm{C}_{2} \mathrm{H}_{4} \mathrm{y} \mathrm{C}_{4} \mathrm{H}_{8}$, por lo que se consideran como una mezcla no polar de compuestos reales. Según los diagramas de selección propuestos por Aspen Technology (Carlson, 1996), para mezclas reales de compuestos no polares, se recomienda usar los siguientes métodos termodinámicos: Soave-Redlich-Kwong (SRK), Peng-Robinson (PR), Lee-Kesler-Plock (LKP), Peng-Robinson-Boston-Mathias (PRBM) y Soave-Redlich-Kwong-Boston-Mathias (SRKBM). 
En la Tabla 5 se presentan los resultados de la comparación de los métodos en el cálculo de las propiedades de la corriente 5 (Figura 1). Las propiedades comparadas fueron los flujos de cada fase, densidad, calor específico y viscosidad dinámica.

Tabla 4 Descripción y parámetros de los controladores del modelo de simulación

\begin{tabular}{|c|c|c|c|c|c|c|}
\hline Lazo & Variable & Variable Manipulada & $\mathrm{K}_{\mathrm{C}}$ & $\mathrm{t}_{\mathrm{I}}$ & $t_{D}$ & Acción \\
\hline Controlada & Variable & Apertura de la válvula & 1.25 & 2 & 0 & Inversa \\
\hline Manipulada & $\mathrm{KC}$ & tI & $\mathrm{tD}$ & $\begin{array}{l}\text { A c - } \\
\text { ción }\end{array}$ & 0 & Inversa \\
\hline FIC-101 & Flujo de ciclohexano & Apertura de la válvula & 1.25 & 2 & 0 & Inversa \\
\hline FIC-102 & Flujo de etileno y buteno & Apertura de la válvula & 1 & 2 & 0 & Inversa \\
\hline FIC-103 & Flujo de vapor a la columna A & Apertura de la válvula & 3.06 & 2 & 0 & Inversa \\
\hline FIC-104 & Flujo de buteno al D-101 & Apertura de la válvula & 0.88 & 2 & 0 & Inversa \\
\hline LIC-101 & Nivel del D-101 & FIC-104 (Cascada) & 11.75 & 6.58 & 1.65 & Inversa \\
\hline PIC-101 & Presión del D-101 & Apertura de la válvula & 55.52 & 1.5 & 0 & Directa \\
\hline FIC-105 & Flujo de líquido a la columna A & Apertura de la válvula & 15.22 & 1.55 & 1.14 & Inversa \\
\hline LIC-103 & Nivel del rehervidor columna A & FIC-105 (Cascada) & 19.41 & 1.25 & 2 & Inversa \\
\hline PIC-102 & Presión de tope de la columna A & Flujo de agua de enfriamiento & 48.02 & 1.95 & 0 & Directa \\
\hline LIC-102 & Nivel del tanque de reflujo columna A & Apertura de la válvula & 3.86 & 8.79 & 2.20 & Inversa \\
\hline FIC-106 & Flujo de fondo de la columna A & Apertura de la válvula & 0.94 & 1.98 & 0 & Inversa \\
\hline LIC-104 & Nivel del tanque de reflujo de la columna B & FIC-106 (Cascada) & 3.72 & 9.02 & 2.25 & Inversa \\
\hline FFIC-101 & Relación de reflujo, columna B & Flujo de reflujo & 1 & 2 & 0 & Inversa \\
\hline PIC-103 & Presión de tope de la columna B & Flujo de agua de enfriamiento & 8.99 & 2.33 & 0 & Directa \\
\hline LIC-105 & Nivel del rehervidor de la columna B & Flujo de vapor al rehervidor & 0.29 & 10.61 & 2.65 & Directa \\
\hline FIC-107 & Flujo de tope de la columna B & Apertura de la válvula & 1.36 & 2.30 & 0 & Inversa \\
\hline FIC-108 & Flujo de fondo de la columna B & Apertura de la válvula & 0.91 & 1.98 & 0 & Inversa \\
\hline FFIC-102 & Relación de reflujo, columna C & Flujo de reflujo & 0.90 & 1.99 & 0 & Inversa \\
\hline FIC-109 & Flujo de tope de la columna C & Apertura de la válvula & 4.55 & 1.99 & 0 & Inversa \\
\hline PIC-104 & Presión de tope de la columna C & Flujo de agua de enfriamiento & 5.64 & 3.70 & 0 & Directa \\
\hline LIC-106 & Nivel del tanque de reflujo de la columna C & FIC-109 (Cascada) & 39.73 & 18.26 & 4.56 & Directa \\
\hline LIC-107 & Nivel del rehervidor de la columna $\mathrm{C}$ & Flujo de vapor al rehervidor & 6.10 & 7.32 & 1.83 & Directa \\
\hline
\end{tabular}


Se puede observar que, de manera general, se obtuvo un ajuste satisfactorio en las propiedades estimadas. Sin embargo, usando los modelos PR, SRK y LKP se obtuvieron errores elevados en el cálculo de la viscosidad de la fase líquida, por lo que se descartó su uso en esta investigación. Los métodos SRKBM y PRBM predicen eficientemente las propiedades del sistema y su uso es recomendado para aplicaciones de refinería, procesamiento de gas y procesos petroquímicos (Al Ghafri, Maitland y Trusler, 2014). Se eligió el método SRKBM, ya que este presenta los menores porcentajes de error en la mayoría de las propiedades estimadas, exceptuando la densidad de la fase vapor.

Tabla 5. Comparación de los modelos termodinámicos

\begin{tabular}{|c|c|c|c|c|c|c|c|c|c|c|}
\hline & \multicolumn{2}{|c|}{ PR } & \multicolumn{2}{|c|}{ SRK } & \multicolumn{2}{|c|}{ LKP } & \multicolumn{2}{|c|}{ SRKBM } & \multicolumn{2}{|c|}{ PRBM } \\
\hline Diseño & Calc. & $\%$ Error & Calc. & $\%$ Error & Calc. & $\%$ Error & Calc. & $\%$ Error & Calc. & $\%$ Error \\
\hline \multicolumn{11}{|c|}{ Fase vapor } \\
\hline \multicolumn{11}{|c|}{ Flujo $(\mathrm{kg} / \mathrm{h})$} \\
\hline 23911.5 & 21877.5 & 8.51 & 23000 & 3.81 & 23421.04 & 2.05 & 24406.08 & 2.07 & 25669.65 & 7.35 \\
\hline \multicolumn{11}{|c|}{ Densidad $\left(\mathrm{kg} / \mathrm{cm}^{3}\right)$} \\
\hline 59.84 & 62.98 & 5.25 & 61.47 & 2.73 & 61.33 & 2.49 & 63.336 & 5.84 & 61.82 & 3.31 \\
\hline \multicolumn{11}{|c|}{ Viscosidad (cP) } \\
\hline 0.014 & 0.013 & 3.63 & 0.014 & 0.53 & 0.013 & 3.64 & 0.014 & 0.40 & 0.014 & 0.41 \\
\hline \multicolumn{11}{|c|}{$\mathrm{Cp}\left(\mathrm{kcal} / \mathrm{kg}^{\circ} \mathrm{C}\right)$} \\
\hline 0.723 & 0.605 & 16.32 & 0.608 & 15.99 & 0.626 & 13.42 & 0.605 & 16.32 & 0.607 & 16.04 \\
\hline \multicolumn{11}{|c|}{ Fase líquida } \\
\hline \multicolumn{11}{|c|}{ Flujo (kg/h) } \\
\hline 67521.76 & 69555.9 & 3.01 & 68433.4 & 1.35 & 68012.35 & 0.73 & 67027.32 & 0.73 & 65763.75 & 2.60 \\
\hline \multicolumn{11}{|c|}{ Densidad $\left(\mathrm{kg} / \mathrm{cm}^{3}\right)$} \\
\hline 544.41 & 528.28 & 2.96 & 528.83 & 2.86 & 529.81 & 2.68 & 522.33 & 4.06 & 462.64 & 15.02 \\
\hline \multicolumn{11}{|c|}{ Viscosidad (cP) } \\
\hline 0.089 & 0.149 & 67.29 & 0.149 & 66.90 & 0.149 & 67.95 & 0.098 & 10.46 & 0.098 & 10.08 \\
\hline \multicolumn{11}{|c|}{$\mathrm{Cp}\left(\mathrm{kcal} / \mathrm{kg}^{\circ} \mathrm{C}\right)$} \\
\hline 0.744 & 0.769 & 3.37 & 0.779 & 4.75 & 0.707 & 4.95 & 0.767 & 3.04 & 0.777 & 4.43 \\
\hline
\end{tabular}




\subsection{Reproducción del balance de masa a partir de los datos de diseño en estado estacionario}

En la Tabla 6 se muestra la comparación de los datos de diseño, con los calculados con el simulador para las corrientes de tope y fondo de la columna A. Se observa que la temperatura y presión calculadas para cada corriente se ajustan adecuadamente a los datos de diseño con errores relativos menores al 2\%; de igual manera para los flujos de cada componente los cuales arrojaron errores menores al $10 \%$.

De manera similar, la comparación entre los flujos de las corrientes de tope y fondo calculados y de diseño de la columna B (Tabla 7), arrojó errores relativos menores al 10\% para todas las variables indicadas, concordando aceptablemente con los datos de diseño.

Comparando las corrientes de tope y fondo de la columna C (Tabla 8), se observa que el modelo reproduce satisfactoriamente la temperatura y presión, así como también para el $\mathrm{C}_{6} \mathrm{H}_{12}$ en el tope y $\mathrm{C}_{11} \mathrm{H}_{24}$ en el fondo, con errores menores al $4 \%$. Sin embargo, el flujo de $\mathrm{C}_{11} \mathrm{H}_{24}$ en el tope de la columna $\mathrm{C}$ es mayor en los datos de diseño, que el reportado por el simulador, posiblemente debido a las diferencias del informe publicado en el balance por diseño la cantidad de $\mathrm{C}_{11} \mathrm{H}_{24}$ a la salida de la columna $\mathrm{C}$.

Tabla 6. Comparación de los flujos de diseño con los calculados con el simulador, para las corrientes de tope y fondo de la columna A

\begin{tabular}{|c|c|c|c|c|c|c|}
\hline \multirow[t]{2}{*}{ Corriente } & \multicolumn{3}{|c|}{ Tope A } & \multicolumn{3}{|c|}{ Fondo A } \\
\hline & Diseño & Simulación & $\%$ Error & Diseño & Simulación & $\%$ Error \\
\hline Temperatura $\left({ }^{\circ} \mathrm{C}\right)$ & 53.4 & 53.4 & 0 & 237 & 237.32 & 0.13 \\
\hline Presión (MPa) & 2.25 & 2.85 & 1.51 & 23.54 & 23.44 & 0.42 \\
\hline Componentes & \multicolumn{2}{|c|}{ Flujo (kg/h) } & \multicolumn{4}{|c|}{ Flujo $(\mathrm{kg} / \mathrm{h})$} \\
\hline $\mathrm{C}_{6} \mathrm{H}_{12}$ & 1000.0 & 1010.32 & 1.03 & 104688,4 & 103869.00 & 0.78 \\
\hline $1-\mathrm{C}_{4} \mathrm{H}_{8}$ & 11051.8 & 10977.70 & 0.67 & 23 & 20.88 & 9.20 \\
\hline 2- $\mathrm{C}_{4} \mathrm{H}_{8}$ & 2940.8 & 2927.47 & 0.45 & 29.5 & 28.84 & 2.24 \\
\hline $\mathrm{C}_{2} \mathrm{H}_{4}$ & 1078.4 & 1075.10 & 0.31 & 0.0 & 0.0 & 0.0 \\
\hline $\mathrm{C}_{11} \mathrm{H}_{24}$ & 0.0 & 0.0 & 0.0 & 298.8 & 295.80 & 1.00 \\
\hline Total & 16086.11 & 16027.87 & 0.36 & 105163.43 & 104319.08 & 0.80 \\
\hline
\end{tabular}


Tabla 7 Comparación de los flujos de diseño con los calculados con el simulador, para las corrientes de tope y fondo de la columna $B$

\begin{tabular}{|c|c|c|c|c|c|c|}
\hline \multirow[t]{2}{*}{ Corriente } & \multicolumn{3}{|c|}{ Tope B } & \multicolumn{3}{|c|}{ Fondo B } \\
\hline & Diseño & Simulación & $\%$ Error & Diseño & Simulación & $\%$ Error \\
\hline Temperatura $\left({ }^{\circ} \mathrm{C}\right)$ & 181 & 182.38 & 0.76 & 185.9 & 187.9 & 1.07 \\
\hline Presión (MPa) & 1.00 & 1.01 & 1.40 & 1.01 & 1.07 & 5.92 \\
\hline Componentes & \multicolumn{2}{|c|}{ Flujo (kg/h) } & \multicolumn{4}{|c|}{ Flujo (kg/h) } \\
\hline $\mathrm{C}_{6} \mathrm{H}_{12}$ & 104599.3 & 103842.0 & 0.72 & 7134.4 & 7119.70 & 0.21 \\
\hline $1-\mathrm{C}_{4} \mathrm{H}_{8}$ & 23.0 & 20.88 & 9.20 & 0.0 & 0.0 & 0.0 \\
\hline $2-\mathrm{C}_{4} \mathrm{H}_{8}$ & 29.5 & 28.84 & 2.24 & 0.0 & 0.0 & 0.0 \\
\hline $\mathrm{C}_{2} \mathrm{H}_{4}$ & 0.0 & 0.0 & 0.0 & 0.0 & 0.0 & 0.0 \\
\hline $\mathrm{C}_{11} \mathrm{H}_{24}$ & 0.0 & 0.0 & 0.0 & 304.8 & 297.88 & 2.27 \\
\hline Total & 104694.33 & 103918.98 & 0.74 & 7575.10 & 7216.18 & 4.74 \\
\hline
\end{tabular}

Tabla 8 Comparación de los flujos de diseño con los calculados con el simulador, para las corrientes de tope y fondo de la columna $C$

\begin{tabular}{|c|c|c|c|c|c|c|}
\hline \multirow[t]{2}{*}{ Corriente } & \multicolumn{3}{|c|}{ Tope C } & \multicolumn{3}{|c|}{ Fondo $\mathrm{C}$} \\
\hline & Diseño & Simulación & $\%$ Error & Diseño & Simulación & $\%$ Error \\
\hline Temperatura $\left({ }^{\circ} \mathrm{C}\right)$ & 144.4 & 144.4 & 0 & 247 & 237.22 & 3.96 \\
\hline Presión (MPa) & 0.49 & 0.5 & 2.04 & 0.52 & 0.54 & 3.05 \\
\hline Componentes & \multicolumn{2}{|c|}{ Flujo (kg/h) } & \multicolumn{4}{|c|}{ Flujo (kg/h) } \\
\hline $\mathrm{C}_{6} \mathrm{H}_{12}$ & 7116.0 & 7173.12 & 0.80 & 18.4 & 26.57 & 44.41 \\
\hline $1-\mathrm{C}_{4} \mathrm{H}_{8}$ & 0.0 & 0.0 & 0.0 & 0.0 & 0.0 & 0.0 \\
\hline $2-\mathrm{C}_{4} \mathrm{H}_{8}$ & 0.0 & 0.0 & 0.0 & 0.0 & 0.0 & 0.0 \\
\hline $\mathrm{C}_{2} \mathrm{H}_{4}$ & 0.0 & 0.0 & 0.0 & 0.0 & 0.0 & 0.0 \\
\hline $\mathrm{C}_{11} \mathrm{H}_{24}$ & 6.0 & 2.30 & 61.61 & 298.8 & 295.57 & 1.08 \\
\hline Total & 7176.60 & 7194.60 & 0.25 & 398.40 & 399.45 & 0.26 \\
\hline
\end{tabular}




\subsection{Análisis de las variables de proceso del área de recuperación de solvente}

Una vez realizada la simulación en estado estacionario y configurados los controladores en el módulo dinámico, se procedió a analizar el comportamiento de los controladores y variables del proceso, luego de interrumpir el suministro de $\mathrm{C}_{2} \mathrm{H}_{4} \mathrm{y} \mathrm{C}_{4} \mathrm{H}_{8}$ en la sección de recuperación de solvente. En la Figura 2 se muestra un descenso rápido del flujo de vapor de alimentación de la columna A, a pesar de que la válvula abre al $100 \%$ no se alcanza el valor deseado, y el flujo se estabiliza en $14100 \mathrm{~kg} / \mathrm{h}$.

De manera similiar ocurre una caída de presión de 0.07 MPa en el D-101, por lo que la válvula se cierra completamente para intentar aumentar la presión. Se nota que el nivel del tanque D-101 (Figura 2.c) aumenta hasta 70\%, lo que causa una disminución del flujo que entra al tambor (Figura 2.d) y poder estabilizar el lazo de nivel. Este aumento se debe a que la válvula de salida del vapor del tanque se cierra completamente.
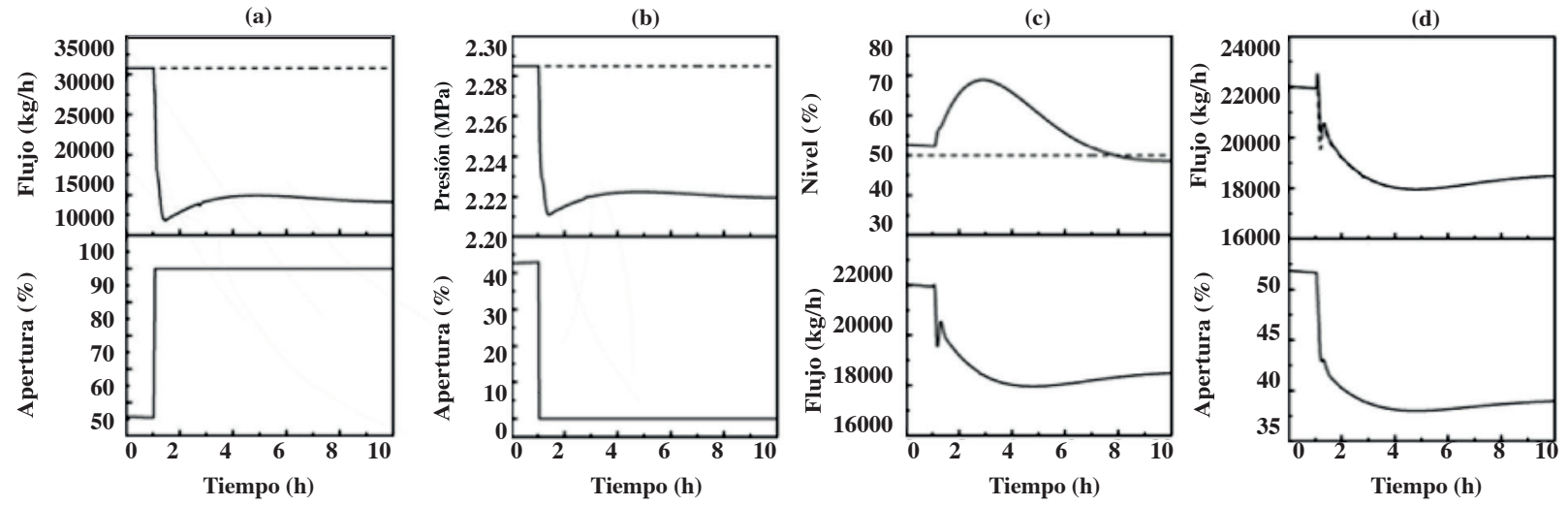

Figura 2. Respuesta de: (a) Flujo de vapor a la columna A, (b) Presión, (c) Nivel y (d) Flujo de entrada del tanque D-101. Variable del proceso: —_, Valor deseado: ---

Por su parte en la Figura 3 se ve que el nivel del rehervidor de la columna A decae completamente, a pesar de abrir la válvula y aumentar el flujo hasta $79500 \mathrm{~kg} / \mathrm{h}$ (Figura 3.b), no fue suficiente para aumentar el nivel en el rehervidor. En cambio, la presión en el tope de la columna A (Figura 3.c) logra estabilizarse disminuyendo el flujo de agua de enfriamiento de 5100 a $3000 \mathrm{~kg} / \mathrm{h}$, debido a la caída de presión. Posteriormente, ocurre un aumento del flujo hasta llegar a $4000 \mathrm{~kg} / \mathrm{h}$ a causa de un aumento de temperatura, como consecuencia de la disminución del nivel del tanque de reflujo de la columna, como se muestra en la Figura 3.d.

Respecto a la Figura 4.a, se presenta el perfil de temperatura de la columna A. Se puede observar un descenso en la temperatura debido a la disminución del flujo de vapor, la cual es más evidente en las primeras etapas de la torre. Luego ocurre un aumento de la temperatura causado por el ajuste de la presión de la torre (Figura 3.b). Seguidamente, se aprecia un segundo aumento de temperatura aproximadamente a las $3 \mathrm{~h}$, como consecuencia del descenso del nivel del tanque de reflujo (Figura 
3.d), hasta estabilizarse en valores desplazados $14{ }^{\circ} \mathrm{C}$ de las condiciones de operación normal para las primeras 11 etapas de la torre.
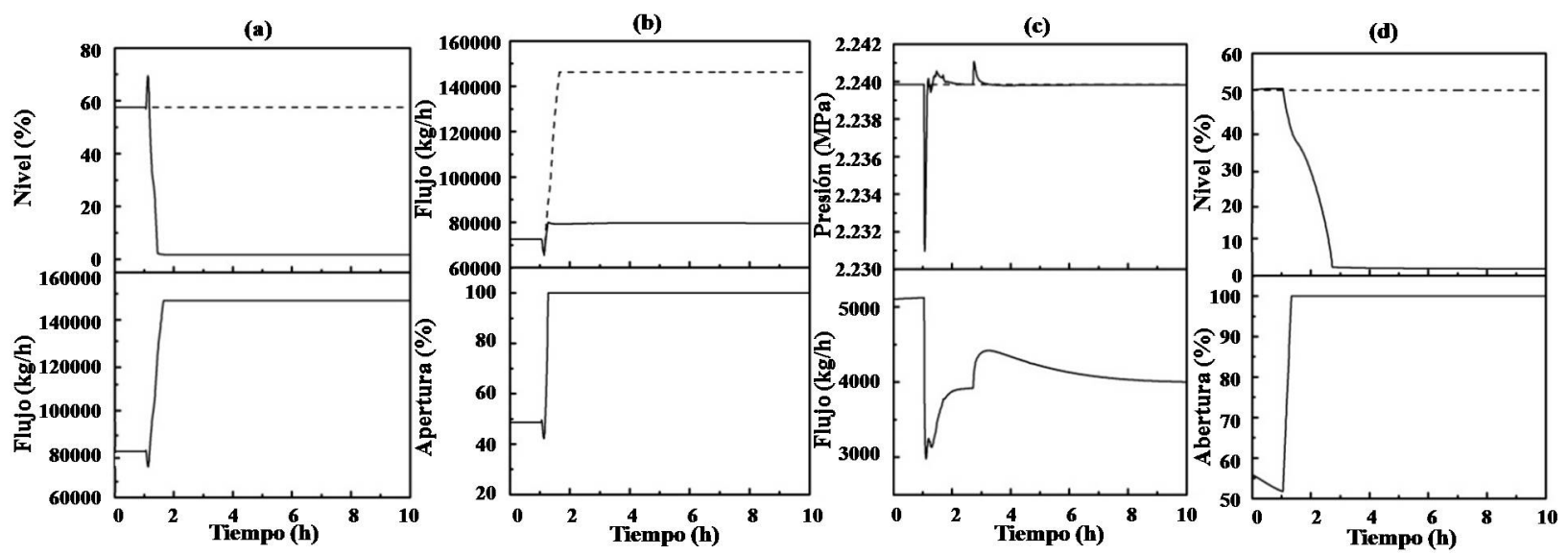

Figura 3. Respuesta de: (a) Nivel del rehervidor, (b) Flujo de líquido, (c) Presión y (d) Nivel del tanque de reflujo de la columna A. Variable del \$proceso: —-, Valor deseado: ---

(a)

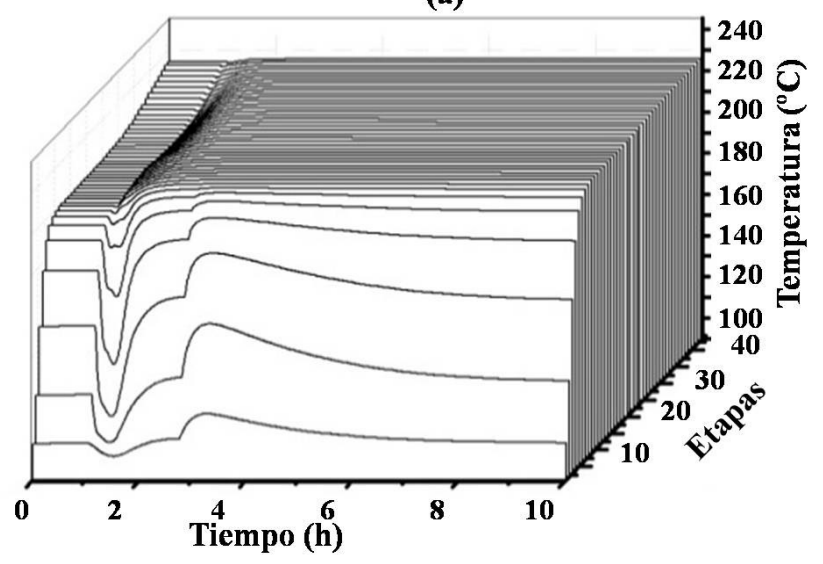

(b)

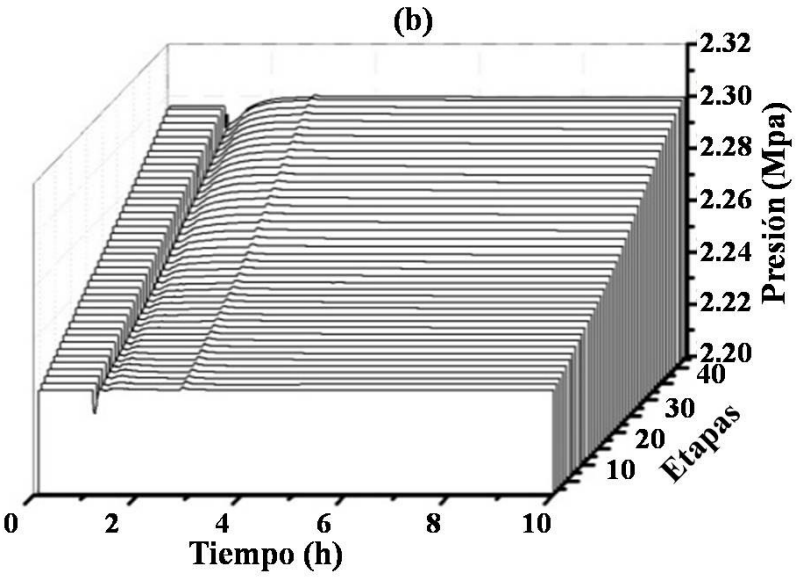

Figura 4. Perfiles de temperatura y presión de la columna A

Análogamente, se presenta en la Figura 4.b el perfil de presión de la columna A. Después de la interrupción del flujo de $\mathrm{C}_{2} \mathrm{H}_{4}$ y $\mathrm{C}_{4} \mathrm{H}_{8}$, dicha presión alcanza un perfil adecuado rápidamente, con un desplazamiento de aproximadamente $0.005 \mathrm{MPa}$ de las condiciones normales de operación de la torre.

En relación a la columna B, se observa en la Figura 5.a que el nivel del tanque de reflujo disminuye lentamente hasta alcanzar el nivel mínimo. El controlador intenta contrarrestar este evento aumentando el flujo de entrada de la columna (Figura 5.b), pero no fue posible alcanzar los valores deseados en ninguno de los controladores. En la Figura 5.c se observa que se logra mantener la relación entre flujo de reflujo y el flujo de alimentación. 
Por otro lado, la presión de la columna B (Figura 5.d) logra estabilizarse en el valor deseado al bajar el flujo de agua de enfriamiento hasta $14500 \mathrm{~kg} / \mathrm{h}$. para compensar la caída de presión. El flujo del tope de la columna B (Figura 6.a), se estabiliza en el valor requerido hasta aproximadamente $4 \mathrm{~h}$, tiempo en el cual el nivel del tanque de reflujo (Figura 5.a) desciende por completo y el flujo llega a bajar a $91500 \mathrm{~kg} / \mathrm{h}$, a pesar de abrir completamente la válvula. El nivel en el rehervidor (Figura 6.b) presenta fluctuaciones a $1 \mathrm{~h}, 2.5 \mathrm{~h}$ y $4.5 \mathrm{~h}$ causadas por la interrupción del flujo de $\mathrm{C}_{2} \mathrm{H}_{4} \mathrm{y} \mathrm{C}_{4} \mathrm{H}_{8}$, por la disminución del nivel del tanque de reflujo de la columna A (Figura 3.d) y del nivel del tanque de reflujo de la columna B (Figura 5.a), respectivamente.
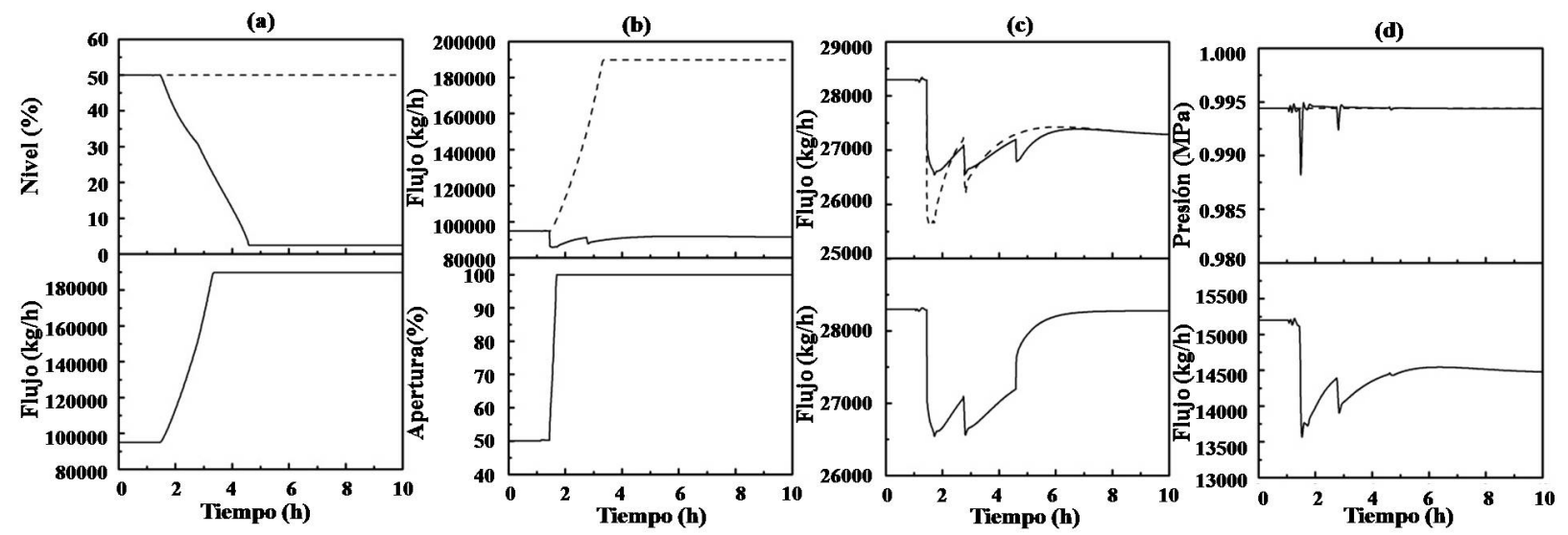

Figura 5. Respuesta de: (a) Nivel del tanque de reflujo, (b) Flujo de entrada, (c) Flujo de reflujo y (d) Presión de la columna B. Variable del proceso: - - Valor deseado: ---
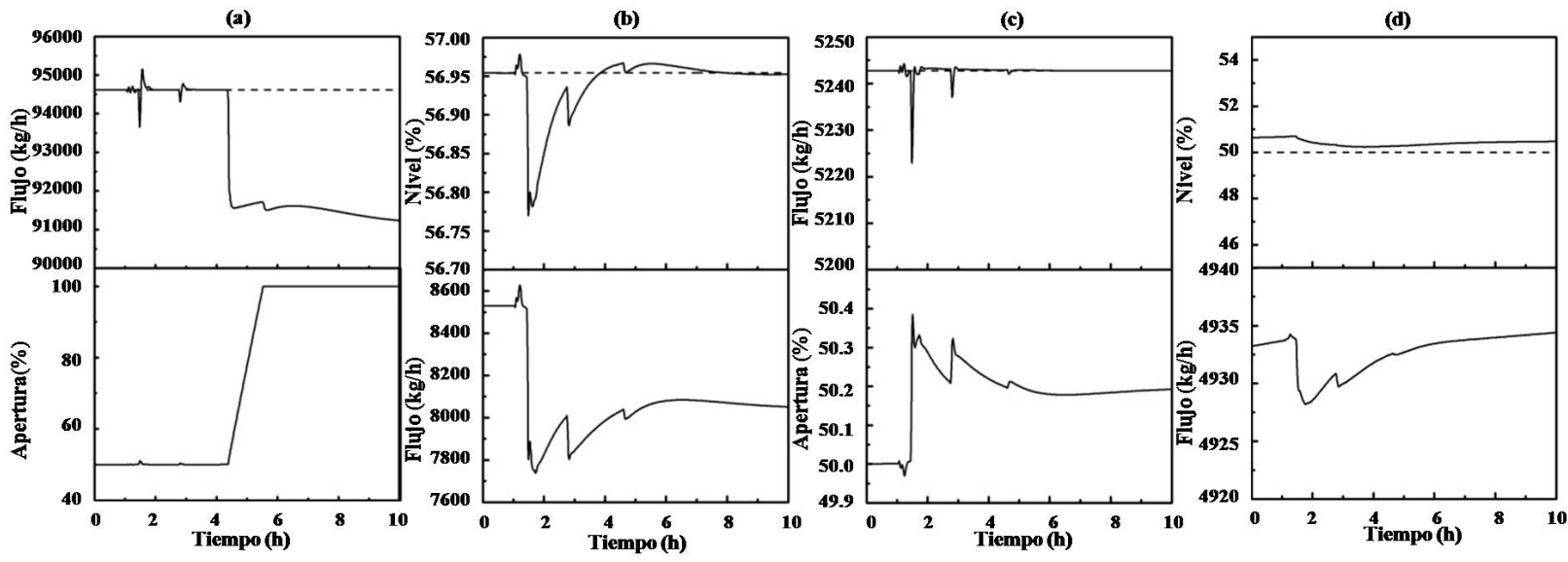

Figura 6. Respuesta de: (a) Flujo de tope y (b) Nivel del rehervidor de la columna B, (c) Flujo de entrada y (d) Nivel del tanque de reflujo de la columna C. Variable del proceso: - - Valor deseado: ---

Ahora bien, en la Figura 7.a se muestra el perfil de temperaturas de la columna B luego de interrumpir el flujo de $\mathrm{C}_{2} \mathrm{H}_{4}$ y $\mathrm{C}_{4} \mathrm{H}_{8}$, se observa que este se estrecha ligeramente, con variaciones de $1^{\circ} \mathrm{C}$ aproximadamente. El perfil de presiones se mantiene constante con una ligera disminución de 
la presión en el fondo de la columna B, como se muestra en la Figura 7.b. Tanto el perfil de temperatura como el de presión se consideran adecuados para operar luego del corte de $\mathrm{C}_{2} \mathrm{H}_{4} \mathrm{y}_{4} \mathrm{H}_{8}$.

(a)

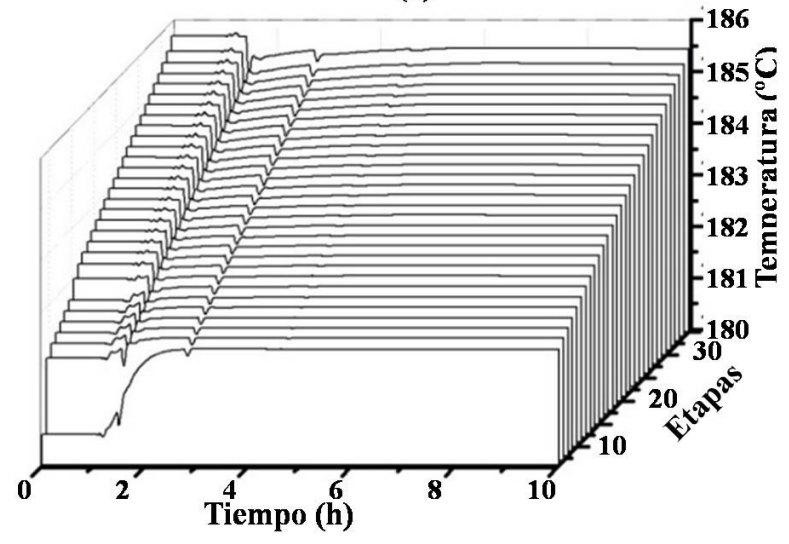

(b)

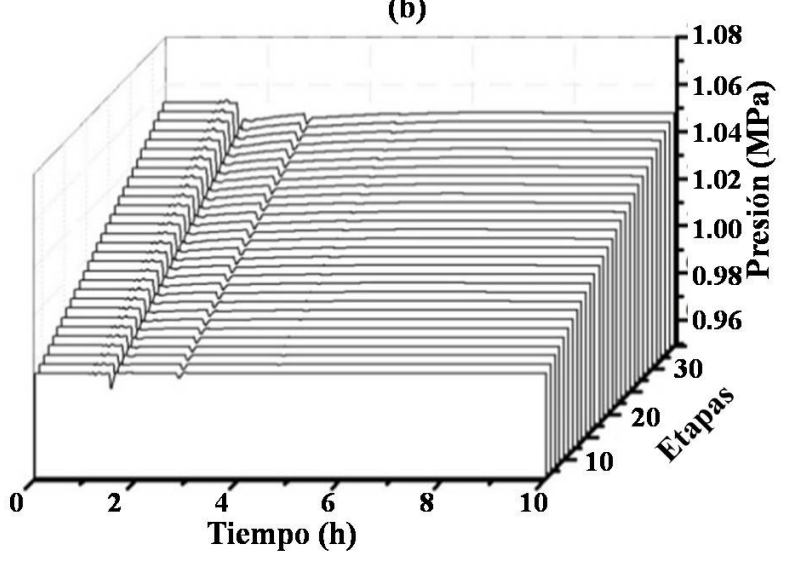

Figura 7. Perfiles de temperatura y presión de la columna B

(a)

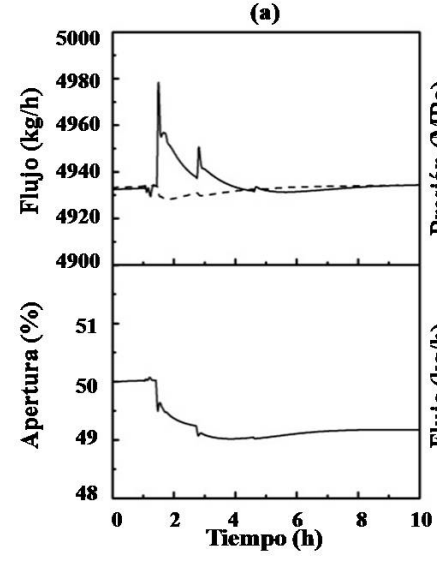

(b)

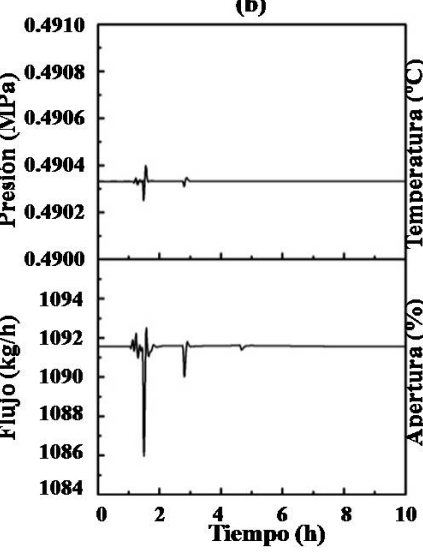

(c)

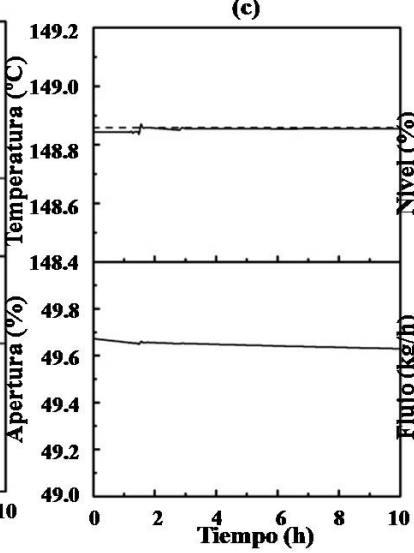

(d)

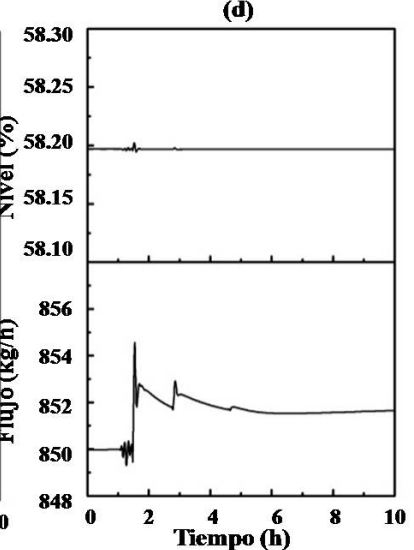

Figura 8. Respuesta de: (a) Flujo de tope, (b) Presión (c) Temperatura de fondo y (d) Nivel del rehervidor de la columna C. Variable del proceso:- - Valor deseado: ---

El flujo de entrada a la columna $\mathrm{C}$ permanece en el valor deseado con leves cambios en la apertura de la válvula (Figura 6.c). El nivel del tanque de reflujo se mantiene igual durante todo el periodo de evaluación (Figura 6.d). Asimismo, el flujo de tope de la columna presenta ligeras perturbaciones mediante la manipulación de la apertura de la válvula, figura 8.a.

Se puede observar que tanto la presión del tope (Figura 8.b) como la temperatura del fondo de la columna $\mathrm{C}$ (Figura 8.c) se mantienen en el valor esperado luego del corte de $\mathrm{C}_{2} \mathrm{H}_{4} \mathrm{y} \mathrm{C}_{4} \mathrm{H}_{8}$. De igual manera, pasa lo mismo con el nivel del rehervidor de la columna C (Figura 8.d). Los perfiles de temperatura y presión de la columna $\mathrm{C}$ se mantienen constante asntes y después de interrumpir el flujo de $\mathrm{C}_{2} \mathrm{H}_{4}$ y $\mathrm{C}_{4} \mathrm{H}_{8}$. 


\subsection{Secuencia de pasos para la adecuación de la sección de recuperación de solvente}

Del análisis de las variables del área de recuperación de solvente, se propusieron distintos pasos para estabilizar los lazos de control y adecuar las condiciones de la sección, con la finalidad de alcanzar rápidamente las condiciones estables una vez se logre restablecer el flujo de $\mathrm{C}_{2} \mathrm{H}_{4}$ y $\mathrm{C}_{4} \mathrm{H}_{8}$.

\subsubsection{Ajuste de la presión de tanque de alimentación D-101 (PIC-101)}

Este controlador en modo automático cierra la válvula completamente para aumentar la presión en el tanque, esto trae como consecuencia que el flujo de vapor de alimentación a la columna A disminuya (Figura 2.b). Al cambiar a modo manual con 50\% de apertura de la válvula, permite que el flujo de vapor llegue a la columna A, a pesar de la caída de la presión del tanque.

\subsubsection{Ajuste del flujo de vapor de alimentación a la columna A (FIC-103)}

Como se mostró en la Figura 2.a, este controlador en modo automático realiza la acción de abrir completamente la válvula para contrarrestar la disminución del flujo de vapor causado por la interrupción del flujo de $\mathrm{C}_{2} \mathrm{H}_{4}$ y $\mathrm{C}_{4} \mathrm{H}_{8}$. En este paso se propone cambiar el modo del controlador a manual con una apertura de la válvula del 50\%, ya que con la apertura de la válvula al 100\% no se puede regular el flujo de vapor que se alimenta a la columna A.

\subsubsection{Ajuste de los lazos de control de nivel}

El tanque de reflujo y rehervidor de la columna A, así como también, el tanque de reflujo de la columna B se vacían completamente a causa de la interrupción del flujo de $\mathrm{C}_{2} \mathrm{H}_{4}$ y $\mathrm{C}_{4} \mathrm{H}_{8}$. En primer lugar se buscó estabilizar el tanque de reflujo de la columna A, manipulando el flujo líquido de alimentación (FIC-105). Para esto, se cambió el modo a manual y se disminuyó la apertura de la válvula hasta que el nivel en el tanque aumente, esto se logró con $47 \%$ de apertura de válvula del controlador FIC-105, valores menores a este porcentaje causan la inundación de tanque de reflujo de la columna A.

Una vez alcanzado el valor deseado, el controlador del nivel del tanque de reflujo regula la entrada del flujo de la corriente 9 (LIC-102) para estabilizar el nivel en 50\%, como se observa en la Figura 9.a. Esto se debe a que al disminuir el flujo de líquido que sale del tanque D-101 aumenta el flujo de vapor que se alimenta a la columna a $19200 \mathrm{~kg} / \mathrm{h}$ además de aumentar la presión del tanque a $2.26 \mathrm{MPa}$.

Similarmente, se propuso disminuir el flujo del producto de fondo de la columna A (FIC-106) para poder estabilizar el nivel del rehervidor, esto se logró al alcanzar el $43 \%$ de apertura de la válvula. Como se muestra en la Figura 10.a el nivel del rehervidor se estabiliza rápidamente en el valor deseado, por lo que el controlador del flujo de entrada de líquido (FIC-105) es capaz de regular el nivel.

Además, se logró asegurar el nivel del tanque de reflujo debido a que el controlador del flujo de alimentación líquida a la columna A se estabiliza al 47\% de apertura de la válvula como en el caso anterior. De igual manera, se propuso disminuir el flujo de salida del tope de la columna B (FIC-107) para ajustar el nivel del tanque de reflujo de la columna B. De este estudio se obtuvo que 
es necesario cerrar la válvula hasta 43\% para ayuda a estabilizar el nivel (Figura 11.a), el lazo de nivel del rehervidor de la columna B permanece estable durante este procedimiento (Figura 11.b).
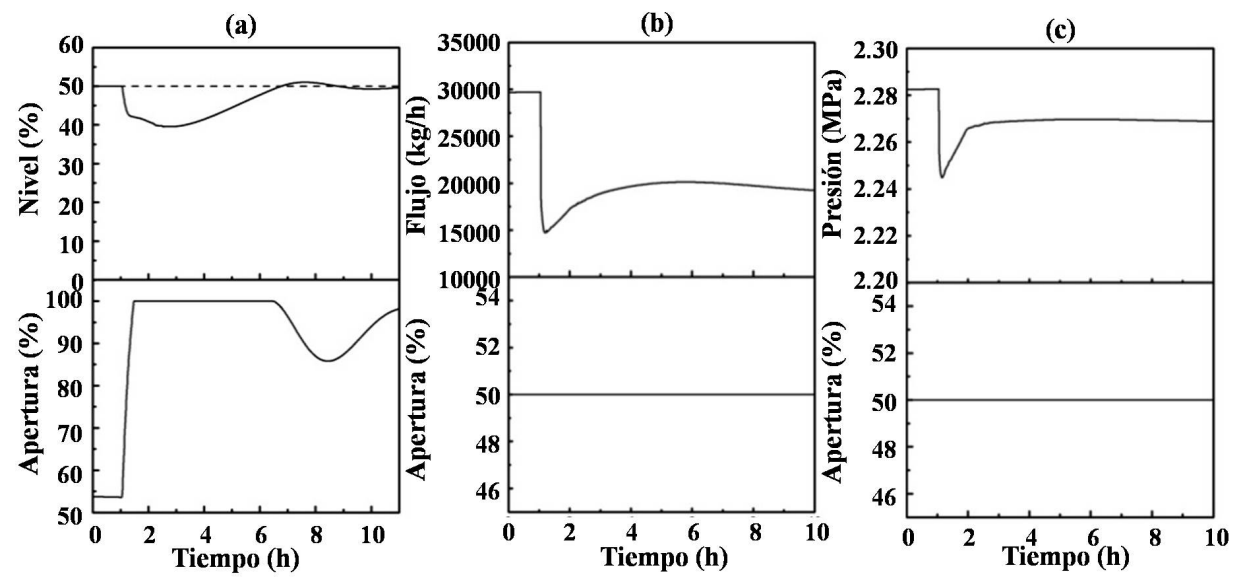

Figura 9. Respuesta de: (a) Nivel del tanque de reflujo de la columna A, (b) Flujo de vapor a la columna A y (c) Presión del D-101 con 47\% de apertura de la válvula de líquido a la columna A

(a)
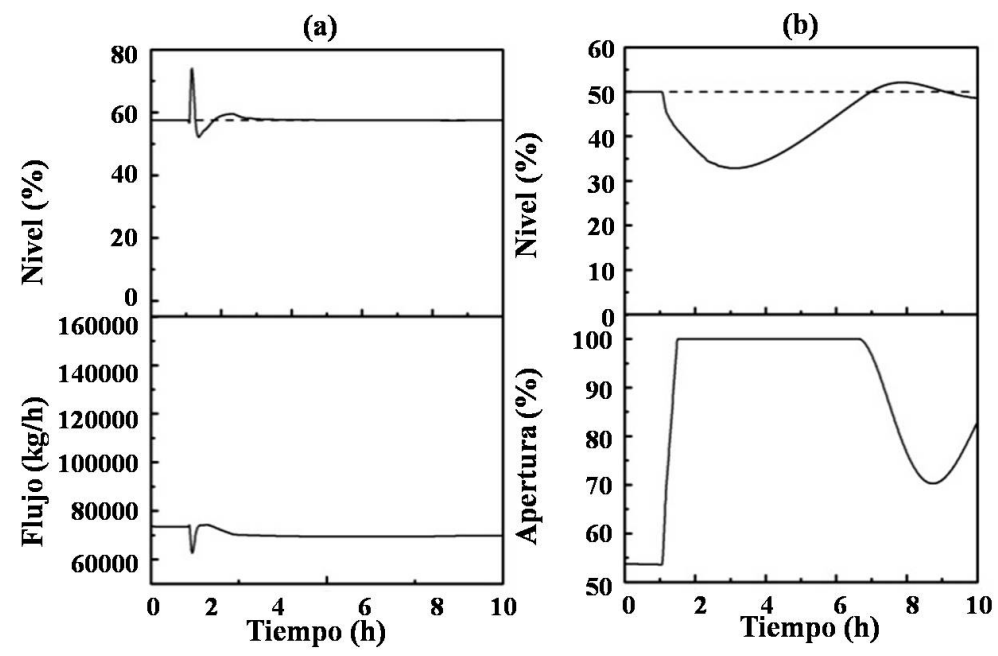

Figura 10. Respuesta del Nivel de (a) Rehervidor y (b)

Tanque de reflujo de la columna A con $43 \%$ de apertura de la válvula del producto de fondo de la columna A

Este paso permitió estabilizar el nivel del rehervidor y tanque de reflujo de la columna A, como se explicó anteriormente, esto se debe a que al disminuir el flujo de salida de la columna B se establece un balance de masa general del proceso que permite la operación de la columna con flujos menores. El ajuste de los lazos de control mediante la manipulación del flujo de producto de tope de la columna B trae como consecuencia un desplazamiento de las temperaturas de los platos a temperaturas mayores (Figura 12.a). El perfil de presión no se vio afectado debido a que los lazos de nivel logran estabilizarse. 
(a)

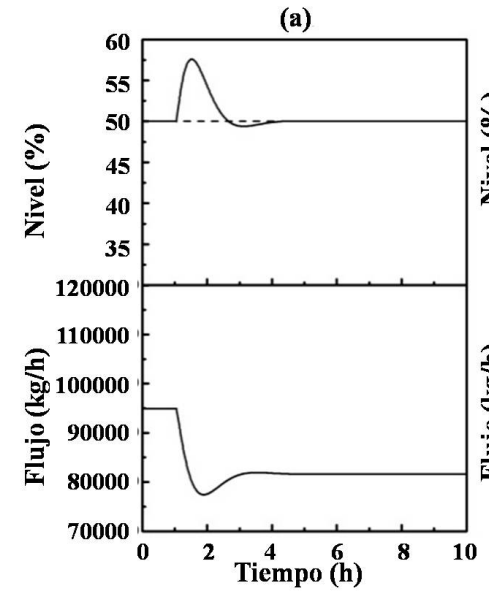

(b)

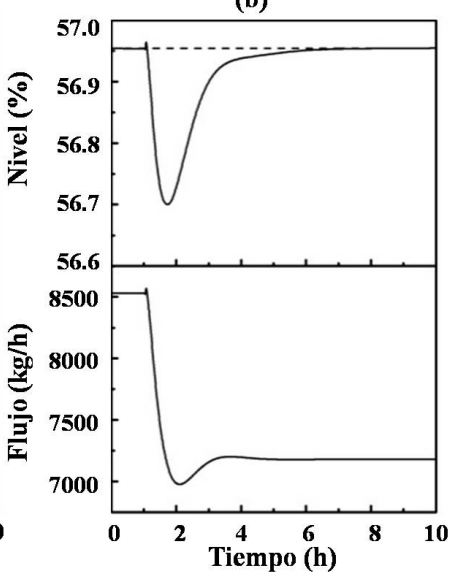

Figura 11. Respuesta del Nivel de (a) Tanque de reflujo y (b) Rehervidor de la columna B con $43 \%$ de apertura de la válvula de producto de tope de la columna B

(a)

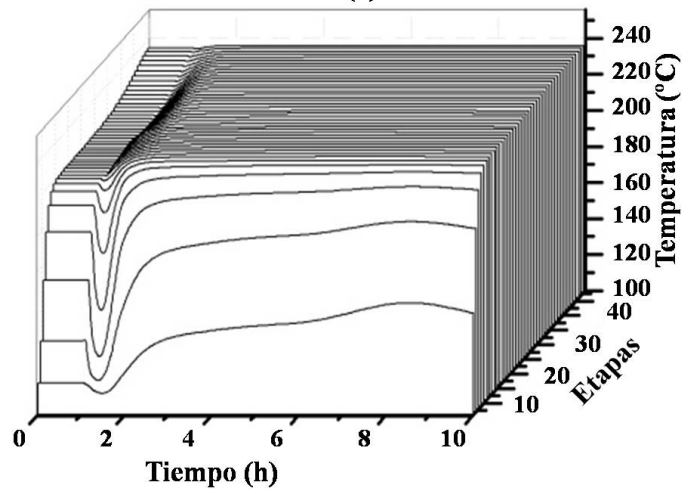

(b)

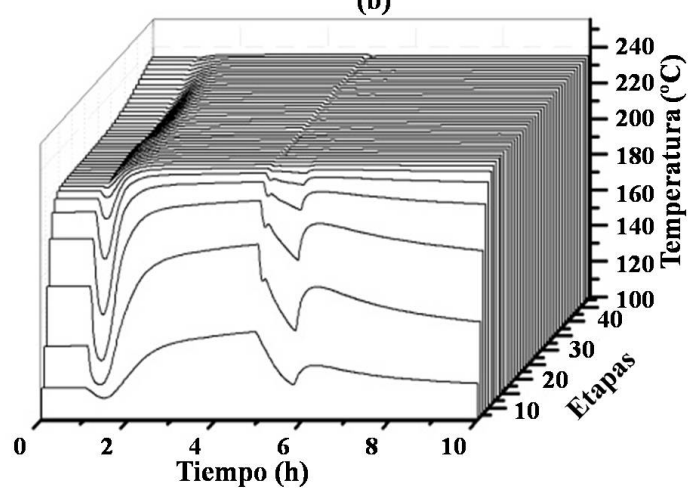

Figura 12. Perfil de temperatura de la columna A con (a) $43 \%$ apertura de la válvula de producto de tope de la columna B y (b) $40000 \mathrm{~kg} / \mathrm{h}$ de flujo de vapor al rehervidor de la columna $\mathrm{A}$

\subsubsection{Ajuste del perfil de temperatura de la columna A}

En este paso se propuso manipular el flujo de vapor al rehervidor de la columna A luego de que se ajustaran los lazos de nivel. Al disminuir este flujo de 52000 a $40000 \mathrm{~kg} / \mathrm{h}$, se lograron estabilizar las temperaturas de la columna a los valores iniciales que corresponden a las temperaturas antes de interrumpir el flujo de $\mathrm{C}_{2} \mathrm{H}_{4}$ y $\mathrm{C}_{4} \mathrm{H}_{8}$. Sin embargo, esta acción trajo como consecuencia que el nivel del tanque de reflujo de la columna A se disminuya completamente. Intentar restablecer este nivel, variando las entradas o salidas de la columna, desplazan el perfil de temperatura nuevamente a valores mayores. 


\subsubsection{Reajuste del nivel tanque de reflujo de la columna A}

El controlador de presión de la columna A (PIC-102) varía el flujo de agua de enfriamiento del condensador para ajustar la presión del tope. Por lo tanto, al cambiar el modo de operación a manual se obtuvo que es necesario aumentar el flujo hasta $5500 \mathrm{~kg} / \mathrm{h}$ para lograr estabilizar el lazo del nivel del tanque de reflujo luego de más de $20 \mathrm{~h}$ de iniciada la prueba como se muestra en la Figura 13. A pesar de estabilizar el nivel, permanece vacío durante dos horas hasta finalmente alcanzar el valor deseado.

La manipulación de los controladores temperatura y presión de la columna A no se incluyen en la secuencia de ajuste de los lazos de control, ya que estos pasos desestabilizan los controladores de nivel lo que puede traer consecuencias negativas en los equipos por falta de flujo, por ejemplo, en la bomba de reflujo de la columna A. Se planteó trabajar con el perfil de temperaturas de la columna A desplazado a mayores temperaturas el cual se restablece una vez restaurado el flujo proveniente del área de reacción. Teniendo en cuenta cada uno de los pasos descritos anteriormente se define como secuencia de ajuste las siguientes acciones: cambio a modo manual de los controladores de flujo de vapor de alimentación a la columna A (FIC-103: 50\% de apertura), presión del D-101 (PIC101: $50 \%$ de apertura) y producto de tope de la columna B (FIC-107: 43\% de apertura).

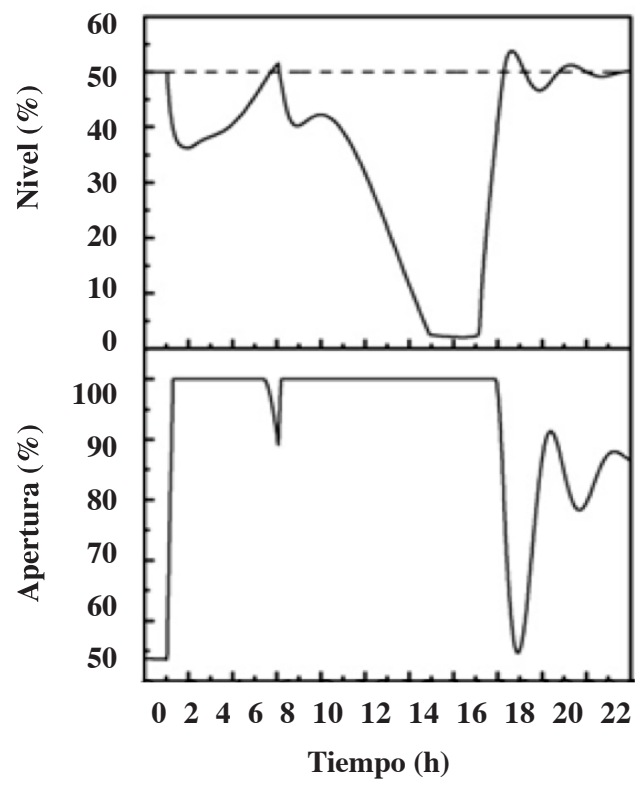

Figura 13. Respuesta del nivel de tanque de reflujo de la columna A con $5500 \mathrm{~kg} / \mathrm{h}$ de flujo de agua de enfriamiento del condensador

Es importante mencionar que el modelo desarrollado en esta investigación se basa en el sistema automático de control actual de la planta de polietileno lineal, razón por la cual se recomienda evaluar una propuesta de sistema de control como alternativa a la actual que pueda compensar más eficientemente las perturbaciones en las variables, así como también un estudio anexo que involucre el área de reacción y el procedimiento de parada de reacción. 


\section{CONCLUSIONES}

La columna A es la más afectada al realizar la interrupción del flujo de $\mathrm{C}_{2} \mathrm{H}_{4}$ y $\mathrm{C}_{4} \mathrm{H}_{8}$, pues se desestabiliza el control del nivel del rehervidor y condensador. El perfil de temperatura aumenta $14{ }^{\circ} \mathrm{C}$ aproximadamente en las primeras etapas de la torre.

En la columna B, solo se ve afectado el nivel del tanque de reflujo, mientras que la columna C logra estabilizarse por sí sola. Se propusieron diferentes acciones para estabilizar los controladores del área de recuperación de solvente: tanto el controlador de flujo de vapor de alimentación a la columna A como el de presión del D-101 se cambian a modo manual con apertura de la válvula en $50 \%$.

Para estabilizar los controladores de nivel se debe cambiar a modo manual el controlador del producto de tope de la columna B con $43 \%$ de apertura de válvula. Ajustar el perfil de temperaturas de la columna A, manipulando el flujo de vapor del rehervidor, desestabiliza los lazos de nivel, estos pueden ajustarse nuevamente manipulando el controlador el flujo de agua de enfriamiento del condensador, pero es un proceso lento que representaría problemas operacionales en la columna A.

\section{REFERENCIAS}

Abdullah,Z., Ahmad, Z. y Aziz, N. (Agosto 2009). MIMO Neural Network Model for Pilot Plant Distillation Column. En 10th International Symposium on Process Systems Engineering, Salvador de Bahía, Brasil.

Al Ghafri, S. Z., Maitland, G. C. y Trusler, J. M. (2014). Experimental and modeling study of the phase behavior of synthetic crude oil $+\mathrm{CO}_{2}$. Fluid Phase Equilibria, 365, 20-40.

Batres, R. (2013). Simulation-based Planning of Shutdown Operations. Procedia Computer Science, 22, 1294-1302.

Carlson, E. (1996). Don't gamble with physical properties for simulations. Chemical Enginering Progress, 20, 36-46.

Fabro, J., Arruda, L. y Neves, F. (2005). Startup of a distillation column using intelligent control techniques. Computers \& Chemical Engineering, 30, 309-230.

Gagieva, S. C., Kurmaev, D. A., Tuskaev, V. A., Zubkevich, S. V., Borissova, A. O., Fedyanin, I. V. et al. (2015). Preparation of linear low-density polyethylene from ethylene by tandem catalysis of titanium(IV) and nickel(II) non-metallocene catalysts. Polyhedron, 98, 131-136.

Hosgor, E., Kucuk, T., Oksal, I. N. y Kaymak, D. B. (2014). Design and control of distillation processes for methanol-chloroform separation. Computers \& Chemical Engineering, 67, 166-177.

Kusolsongtawee, T. y Bumroongsri, P. (2017). Optimización of energy consumption in gas-phase polymerization process for linear low density polyethylene production. Energy Procedia, 138, 772-777.

Leitao, P., Colombo W. y Karnouskos S. (2015). Industrial automation based on cyber-physical systems technologies: Prototype implementations and challenges. Computers in Industry, 35 (8), 11-25. 
Luyben, W. (2012). Use of dynamic simulation for reactor safety analysis. Computers \& Chemical Engineering, 40, 97-109.

Luyben, W. (2013). Distillation design and control using AspenTM Simulation. Nueva York: John Wiley \& Sons, Inc.

Malpass, D. (2010). Introduction to Industrial Polyethylene: Properties, Catalysts, and Processes. Nueva York: John Wiley \& Sons, Inc.

Niculescu, A., Constantin, T., Ana, G. y Draghia, M. (2017). Dynamic simulation of a multicomponent distillation column for D-T separation. Fusion Engineering and Design, 124, 752-756.

Sabetzadeh, M., Bagheri, R. y Masoomi, M. (2016). Effect of nanoclay on the properties of low density polyethylene/linear low density polyethylene/thermoplastic starch blend films. Carbohydrate Polymers, $141,75-81$.

Taqvi, S. A., Tufa, L. D. y Muhadizir, S. (2016). Optimization and Dynamics of Distillation Column Using Aspen Plus®. Procedia Engineering, 148, 978-984.

Whiteley, K. (2012). Ullmann's Encyclopedia of Industrial Chemistry. Nueva York: John Wiley \& Sons, Inc.

Xu, S. y Bao, J. (2017). Distributed control of plantwide chemical processes. Journal of Process Control, 19 (10), 1671-1687.

Ziegler, J. G. y Nichols, N. B. (1993). Optimum settings for automatic controllers. Journal of Dynamic Systems, Measurement, and Control, 115, 220-222. 